\title{
Optical MEMS Pressure and Vibration Sensors Using Integrated Optical Ring Resonators
}

\author{
Prasant Kumar Pattnaik, Bh. Vijayaaditya, T. Srinivas and A. Selvarajan \\ Department of Electrical Communication Engineering \\ Indian Institute of Science, Bangalore 560012, India. \\ Tel: +91-80-2293 2852, Fax: +91-80-2360 0563 \\ Email: pattu@ece.iisc.ernet.in
}

\begin{abstract}
In this paper we propose and analyse novel optical MEMS based pressure and vibration sensors utilising integrated optical ring resonators. The wavelength shift of light propagating in the resonator, located over the micro-mechanical structure, due to stress induced refractive change because of applied pressure or vibration, provides the desired sensor output. In the case of pressure sensor, as the circular diaphragm deflects due to the differential pressure, stress induced refractive index change in the waveguide lead to change in the wavelength shift providing the measure of pressure. For $1 \mathrm{~mm}$ radius circular diaphragm with $65 \mu \mathrm{m}$ thickness, wavelength shift of $0.78 \mathrm{pm} / \mathrm{kPa}$ is obtained with a pressure range of $300 \mathrm{kPa}$. In the case of vibration sensor, the straight portion a race track resonator is located at the foot of a cantilever beam with proof mass. As the beam deflects due to vibration, stress induced refractive change in the waveguide located over the beam lead to the wavelength shift providing the measure of vibration. A wavelength shift of $3.19 \mathrm{pm} / \mathrm{g}$ in the range of $280 \mathrm{~g}$ for a cantilever beam of $1750 \mu \mathrm{m} \times \mathbf{4 5 0} \mu \mathrm{m} \times 20 \mu \mathrm{m}$ has been obtained. Since the wavelength of operation is around 1.55 $\mu \mathrm{m}$, hybrid integration of source and detector is possible on the same substrate Also it is less amenable to noise as wavelength shift provides the sensor signal. This type of sensors can be used for precession instrumentation, aerospace application and other harsh environments with suitable design.
\end{abstract}

\section{INTRODUCTION}

Micro-opto-electro-mechanical (MOEM) devices and systems, based on the principles of integrated optics and micromachining technology on silicon have immense potential for sensor applications [1]. Optical MEMS sensors have the advantages of immunity to EMI, larger bandwidth and higher sensitivity compared to electronic counter parts. Many types of optical MEMS pressure sensors using Mach-Zehnder interferometer(MZI) [2], [3], Fabry-Perot interferometer [4] have been reported. Phase change in the sensing arm of MZI due to applied pressure is read out as intensity change in case of MZI baed sensor. In Fabry-Perot interferometer based pressure sensor, the variation of cavity length because of displacement of the diaphragm due to applied pressure is readout as intensity changes. Brabander et.al. [5] have considered ring resonator based pressure sensor whose straight arms are over the long edge of a micromachined rectangular diaphragm. But here we consider a circular silicon diaphragm and consider the resonant wavelength shift of the ring resonator as a measure of applied differential pressure.

Optical MEMS vibration sensors utilise the advantages of optics to get better sensitivity with the integration and minia- turisation capabilities of MEMS. Few optical MEMS vibration sensors have been reported in the literature in contrast to many types of MEMS vibration sensors [6]. Most of the optical MEMS vibration sensors utilise the optical power transfer between the waveguide on the cantilever beam to the waveguide on the substrate due to the vibrating force [7]-[9]. Since the waveguide core sizes are small, the power variations is small and is useful over a small dynamic range. Directional coupler based optical MEMS vibration sensor was reported by us earlier [10]. But this uses the intensity measurement and more amenable to noise.

In this paper we report novel optical MEMS pressure and vibration sensor based on the wavelength shift of optical resonator located over the micromechanical sensing element. Ring resonator over circular diaphragm for pressure sensor and race track resonator with its straight portion over the cantilever beam for the vibration sensor are considered here.

\section{OptiCAL MEMS PRESSURE SENSOR}

Fig. 1 shows the schematic of the optical MEMS pressure sensor. When pressure is applied on the diaphragm, due to

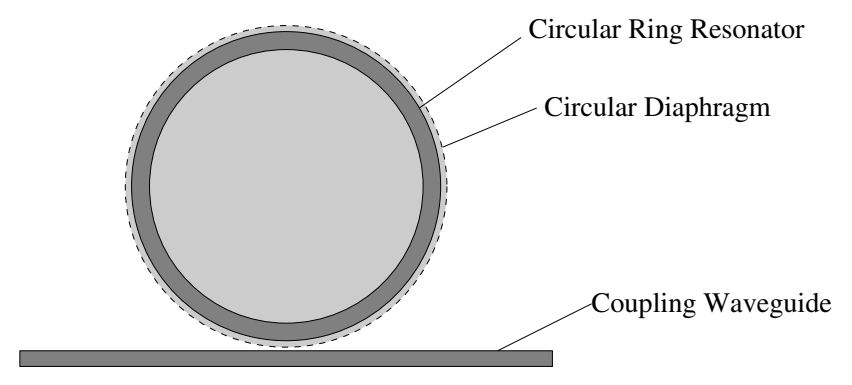

Fig. 1. Schematic of the proposed Optical MEMS pressure sensor

the stress in the waveguide located over the diaphragm, the refractive index changes (due to elasto-optic effect) and hence the phase of the light propagating through it. This phase change is read out as resonant wavelength shift of the ring resonator located over the diaphragm. Since the waveguide is along the edge of the diaphragm where maximum stress occurs, phase change in the waveguide due to cumulative photo-elsatic effect is more. The design of the sensor involves the design of mechanical diaphragm, optical ring resonator and 
opto-mechanical coupling between them for sensor readout as wavelength shift.

\section{A. Ring Resonator}

The ring resonator is located over the edge of the diaphragm as shown in Fig. 1. Light is coupled into and out of the ring from the straight waveguide placed adjcent to it over the substrate. The ring is made up of oxinitride $\left(\mathrm{SiO}_{2} / \mathrm{SiON} / \mathrm{SiO}_{2}\right)$ waveguide single moded at $1.55 \mu \mathrm{m}$. The ratio of the output and input field $\left(I_{t}\right)$ is given by [11]

$$
I_{t}=\left[\frac{\left(1-k^{2}\right)^{\frac{1}{2}}-\exp \left\{-\left(\alpha_{T}+i \phi\right)\right\}}{1-\left(1-k^{2}\right)^{\frac{1}{2}} \exp \left\{-\left(\alpha_{T}+i \phi\right)\right\}}\right]^{2}
$$

where, $k$ is the coupling coefficient, $\alpha_{T}=\alpha L$ is the total propagation loss in the ring, $\phi=2 \pi n_{e f f} L / \lambda$ and $L=$ $2 \pi r$, where $r$ is the radius of the ring. Fig. 2 shows the

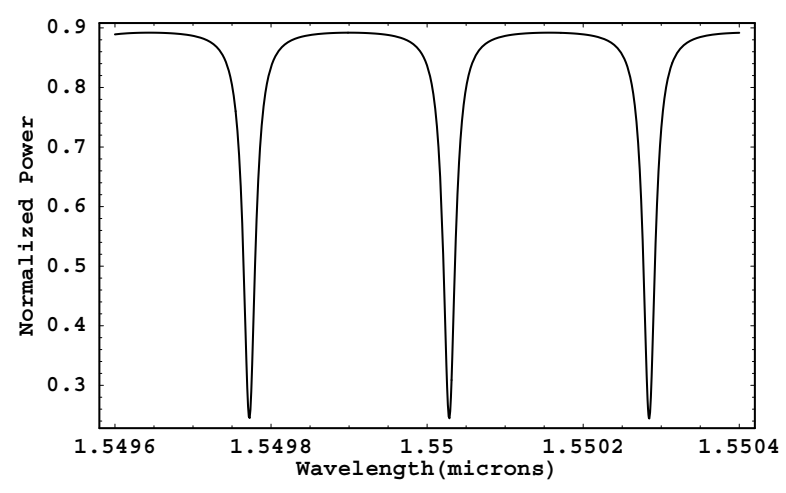

Fig. 2. Output characteristics of the ring resonator

intensity variations at the output with the variation in the input wavelength with periodic dips at resonant wavelengths. Here $\mathrm{r}=1 \mathrm{~mm}$ and $n_{\text {ef } f}=1.48789$ at $1550 \mathrm{~nm}$ is taken for the desired waveguide structure with $\mathrm{k}=0.1 \%$ and very low loss $(\alpha \simeq 0)$. As seen from the graph, the free spectral range (FSR) is about $257 \mathrm{pm}$.

\section{B. Mechanical Design and Opto-mechanical Coupling}

The deflection $w$ of a circular diaphragm of radius ' $a$ ' and thickness $\mathrm{h}$ due to differential pressure $\mathrm{P}$ is given by

$$
D \nabla^{4} w(r)=P
$$

along with the built-in edge boundary conditions $w(a)=0$, w' $^{\prime}(\mathrm{a})=0$ and $\mathrm{w}^{\prime}(0)=0$, where $D=E h^{3} /\left(12\left(1-\nu^{2}\right)\right)$ is the flexural rigidity, $E$ is the Young's modulus and $\nu$ is the Poisson's ratio. The radial and tangential stresses at the surface of the circular diaphragm $(z=h / 2)$ can be obtained as

$$
\begin{aligned}
& \sigma_{r}=\frac{3 a^{2}}{8 h^{2}} P\left\{(3+\nu) \frac{r^{2}}{a^{2}}-(1+\nu)\right\} \\
& \sigma_{t}=\frac{3 a^{2}}{8 h^{2}} P\left\{(1+3 \nu) \frac{r^{2}}{a^{2}}-(1+\nu)\right\}
\end{aligned}
$$

The maximum radial stress occurs at the edge of the circular diaphragm and is $\frac{3 a^{2}}{4 h^{2}} P$. Hence the waveguide is placed at the edge to get the maximum refractive index change due to photoelastic effect. Larger radius and thinner diaphragm will have more stress for a given applied pressure. The radius of the diaphragm is chosen considering the ring radius which dictates the resonant wavelengths.

Due to the stress, the refractive index of the waveguide changes. Refractive index change is related to the stress through a fourth rank photo-elastic tensor. The refractive index change in the present case is given by

$$
\Delta n=C \sigma_{r}
$$

where $C=4.22 \times 10^{-12} / \mathrm{Pa}$ for the oxynitride core waveguide. Fig. 3 shows the normalised refractive index change $\left(P a^{2} h / D\right)$ along the diameter. Maximum index change occur

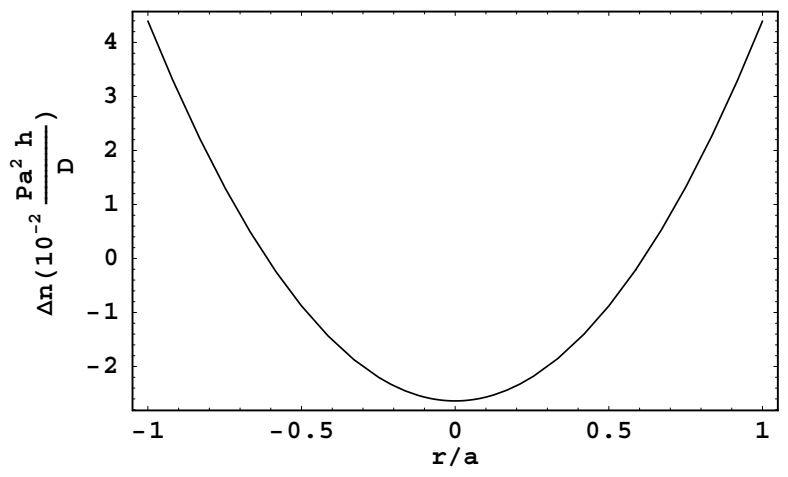

Fig. 3. Normalised refractive index change $\left(\frac{P a^{2} h}{D}\right)$ along the diameter of the diaphragm

at the edge of the diaphragm since the stress is more there and hence the resonator waveguide is located there.

\section{Sensor Readout}

The cumulative phase change along the edge of the diaphragm is given by

$$
\Delta \phi(P)=\frac{2 \pi}{\lambda}\left(\frac{n}{n_{e f f}}\right) C\left(\frac{3 a^{2}}{4 h^{2}}\right)(2 \pi a) P
$$

The phase sensitivity here is about $19 \mu \mathrm{rad} / \mathrm{Pa}$ for diaphragm thickness of $65 \mu \mathrm{m}$. Since maximum allowable phase change can be $2 \pi$, about $300 \mathrm{kPa}$ can be detected. The wavelength shift due to applied pressure can be written as

$$
\Delta \lambda_{\text {Shift }}=\Delta \lambda_{F S R} \frac{\Delta \phi(P)}{2 \pi}
$$

where $\Delta \lambda_{F S R}=\lambda^{2} /\left(2 \pi n_{e f f} a\right)$ is the free spectral range (FSR) in terms of wavelength. Fig. 4 shows the variation of the intensity versus input wavelength for an applied pressure of $100 \mathrm{kPa}$. The dotted curve shows the variations without any applied pressure. It is observed from the graph that there is a resonant wavelength shift of $78 \mathrm{pm}$ for $100 \mathrm{kPa}$. Linear variation of wavelength shift $(0.78 \mathrm{pm} / \mathrm{kPa})$ in the range of 300 $\mathrm{kPa}$ can be achieved with this design. In the present design, the diaphragm thickness is taken to be $65 \mu \mathrm{m}$. However by reducing the thickness (e.g $10 \mu \mathrm{m}$ ), one can achieve better sensitivity $(32 \mathrm{pm} / \mathrm{kPa})$, for low pressure $(7.5 \mathrm{kPa})$ sensing applications. 


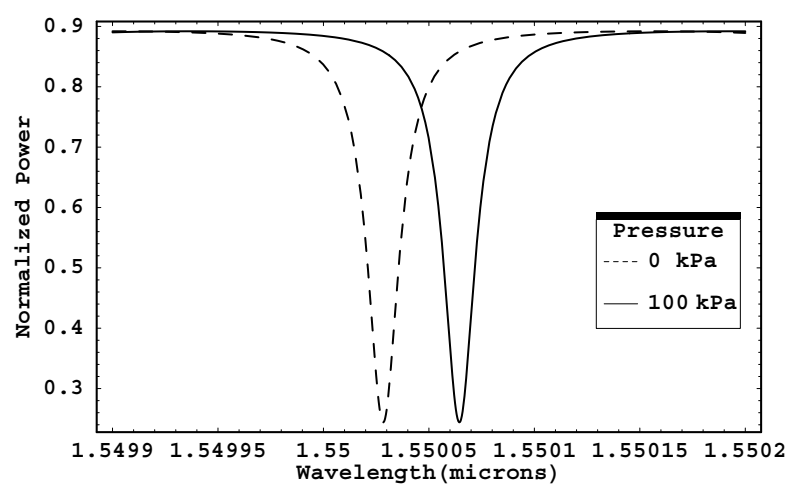

Fig. 4. Intensity vs Wavelength for 0 and $100 \mathrm{kPa}$

\section{OPTICAL MEMS VIBRATION SENSOR}

As in the case on optical MEMS pressure sensor, optical MEMS vibration sensor too can be based on a resonating optical geometry such as ring resonator. Since the length dimension is longer than the width in the mechanical structure (cantilever beam) of the vibration sensor, it is difficult to use the ring resonator. But race track resonator can effectively be used in this case. Fig. 5 shows the schematic of proposed vibration sensor using racetrack resonator. As the cantilever

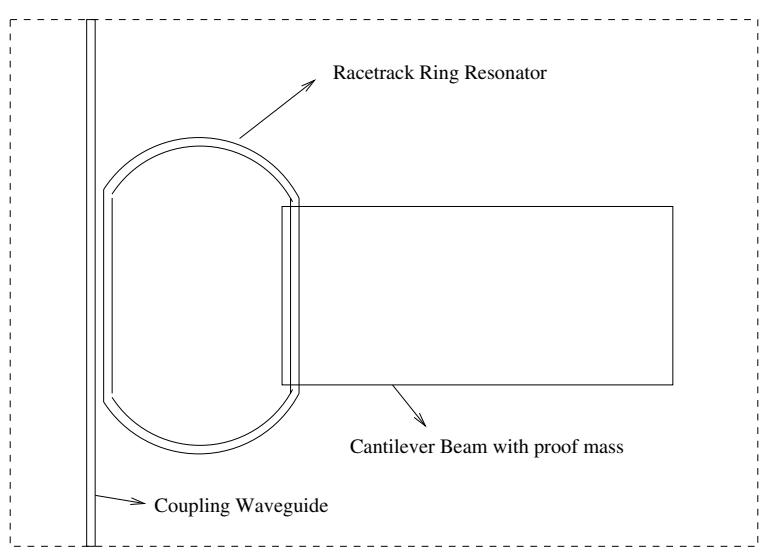

Fig. 5. Schematic of proposed optical MEMS vibration sensor using racetrack resonator

beam deflects due to vibration, the stress induced refractive index change produces phase change in the waveguide located over the foot of the beam and hence the vibration is readout as resonant wavelength shift of the racetrack resonator. The design of the sensor involves the mechanical design of cantilever beam, racetrack resonator and opto-mechanical coupling for the sensor readout as wavelength shift.

\section{A. Racetack Resonator}

The straight portion of the racetrack resonator is taken to be the width $(b)$ of the cantilever beam as shown in Fig. 5. The length of the curved path, for which the line joining the arc is same as width of the beam, is given by

$$
L=2 r \sin ^{-1}\left(\frac{b}{2 r}\right)
$$

where $r$ is the radius of curvature of the arc. The arc and the straight waveguide are assumed to have continuity without sharp bends. The total optical path length of the resonator is

$$
L_{o p}=2 n_{e f f}\left\{b+2 r \sin ^{-1}\left(\frac{b}{2 r}\right)\right\}
$$

The FSR of this resonator is given by $c / L_{o p}$. The resonance characteristics is determined by the total optical length dictated by the $b, r$ and effective refractive index of the waveguide. The curved portion is taken as part of the circle of $1 \mathrm{~mm}$ radius (since oxynitride waveguide can have at best $1 \mathrm{~mm}$ bend radius without much loss [12]) with angle 0.454 rad such that the straight line joining the arc is $450 \mu \mathrm{m}$ (the cantilever beam width). The FSR of this resonator is $111.5 \mathrm{GHz}(893 \mathrm{pm})$.

\section{B. Mechanical Design and Opto-mechanical Coupling}

The mechanical element in vibration sensor is a cantilever beam with length $l_{1}$ and a proof mass of sides $l_{2}$ with thickness $t_{w}$ (wafer thickness). This can be modeled as a cantilever beam of length $\left(l=l_{1}+l_{2} / 2\right)$ with proof mass $\mathrm{m}$ attached at the center of $l_{2}$ (valid for $l_{2} \geq 10 l_{1}$ [13]) satisfying

$$
m \frac{d^{2} w}{d t^{2}}+c \frac{d w}{d t}+k w=q
$$

with spring constant $k=3 E I_{1} / l^{3}$ where $I_{1}=b h^{3} / 12$ is the moment of inertia of the beam. The fundamental frequency is given by

$$
f=\frac{1}{2 \pi} \sqrt{\frac{3 E I_{1}}{m\left(l_{1}+l_{2} / 2\right)^{3}}} .
$$

The damping coefficient $\mathrm{c}$ and the fundamental frequency dictate the mechanical dimensions of the beam. Normalised damping coefficient $(\xi=c / 2 m \omega)$ is adjusted to 0.7 to get maximum linearity. For $l_{1}=250 \mu \mathrm{m}, l_{2}=3000 \mu \mathrm{m}, \mathrm{b}=450 \mu \mathrm{m}$, $\mathrm{h}=20 \mu \mathrm{m}$, fundamental frequency is $410 \mathrm{~Hz}$ and the required gap for $\xi=0.7$ is $33 \mu \mathrm{m}$. The bending stress (in units of $\mathrm{g}$ ) at the foot of the cantilever and on the top surface is now given by

$$
\sigma_{y y}=\frac{m g\left(l_{1}+l_{2} / 2\right)}{6 b h^{2}} .
$$

For beam with $l_{2}=3000 \mu \mathrm{m}$, the stress is $2.46 \mathrm{MPa} / \mathrm{g}$. Since the fracture stress of the material(silicon) is $7 G P a$, maximum acceleration (breakdown) for these dimensions is $2900 \mathrm{~g}$ when a safety factor of 2 is taken into account.

The change in refractive index of the waveguide located at the foot of the beam is given as earlier $\Delta n=C \sigma_{y y}$. Since the index change is uniform over the waveguide as the stress is constant across the width, the phase change in the waveguide in terms of beam parameters(in units of $\mathrm{g}$ ) is given by $\Delta \phi=$ $k_{\phi} g$, where

$$
k_{\phi}=\frac{2 \pi}{\lambda} \frac{n}{n_{e f f}} C \frac{6 m\left(l_{1}+\frac{l_{2}}{2}\right)}{h_{1}^{2}} .
$$


Phase change is more for longer and thinner beam. For $l_{2}=3000 \mu \mathrm{m}$ and $h_{1}=20 \mu \mathrm{m}$, phase change is $22.4 \mathrm{mrad} / \mathrm{g}$.

\section{Sensor Readout}

As in pressure sensor case, the phase change produces wavelength shift is $\Delta \lambda_{\text {Shift }}=\Delta \lambda_{F S R} \Delta \phi(g) /(2 \pi)$, where $\Delta \lambda_{F S R}$ is the FSR in terms of wavelength. The vibration amplitude in units of $g$ is readout as this wavelength shift. For the chosen dimensions, wavelength shift of $3.19 \mathrm{pm} / \mathrm{g}$ occurs. Fig. 6 shows the wavelength shift for $70 \mathrm{~g}$ vibration amplitude with respect to the original resonating wavelength. Since the



Fig. 6. Wavelength shift with and without applied acceleration of $70 \mathrm{~g}$ for optical MEMS vibration sensor

FSR is 893 pm, upto $280 \mathrm{~g}$ vibration amplitude with $410 \mathrm{~Hz}$ vibration frequency can be measured.

\section{CONCLUSION}

We have proposed and analysed novel optical MEMS pressure and vibration sensor using integrated optical ring resonators. Ring resonator over the edge of circular diaphragm for pressure sensor and racetrack resonator with straight portion over the edge of cantilever beam for the vibration sensor are analysed. Wavelength shift due to the pressure or vibration produce the desired output. For $1 \mathrm{~mm}$ radius circular diaphragm, wavelength shift of $0.78 \mathrm{pm} / \mathrm{kPa}$ is obtained in a pressure range of $300 \mathrm{kPa}$. A wavelength shift of $3.19 \mathrm{pm} / \mathrm{g}$ in the range of $280 \mathrm{~g}$ for a cantilever beam of $1750 \mu \mathrm{m} \times 450 \mu \mathrm{m} \times 20$ $\mu \mathrm{m}$ has been obtained. Because the frequency of operation is around $1.55 \mu \mathrm{m}$, hybrid integration of source and detector is possible on the same substrate. Also since the detection is in frequency domain, it is less amenable to noise. With suitable modification in the design parameters, optical MEMS pressure sensors can be used for blood pressure monitoring, precession instrumentation, aerospace propulsion application and other harsh environments and optical MEMS vibration sensor can be used for machinery vibration, geophysical sensing and electromagnetically harsh environments.

\section{ACKNOWLEDGMENTS}

One of the authors (P. K. Pattnaik) wish to acknowledge Council of Scientific and Industrial Research (CSIR) and Department of Science and Technology (DST), Govt. of India for providing travel grants to attend the conference. One of the authors (T. Srinivas) wish to thank Indian Institute of Science for providing financial assistance to attend the conference.

\section{REFERENCES}

[1] M. Tabib-Azar and G. Behelm, "Modern trends in microstructures and integrated optics for communication, sensing and signal processing," Opt. Engg., vol. 36, no. 5, pp. 1307-1318, May 1997.

[2] M. Ohkawa, M. Izustu, and T. Sueta, "Integrated optic pressure sensor on silicon substrate," Appl. Opt., vol. 28, no. 23, pp. 5153-5157, Dec. 1989.

[3] P. K. Pattnaik, A. Selvarajan, and T. Srinivas, "Guided wave optical MEMS pressure sensor," in Proc. of ISA/IEEE Conference on Sensors for Industry (SIcon/05), Houston, Texas, USA, Feb. 2005, pp. 122-125.

[4] Y. Kim and D. P. Neikrik, "Micromachinded fabry-perot cavity pressure sensor," IEEE Photon. Technol. Lett., vol. 7, no. 12, pp. 1471-1473, Dec. 1995.

[5] G. N. D. Brabender, J. T. Boyd, and G. Beheim, "Integrated optical ring resonator with micromechanical diaphragm for pressure sensing," IEEE Photon. Technol. Lett., vol. 6, no. 5, pp. 671-673, May 1994.

[6] J. Bernstein, R. Miller, W. Kelly, and P. Ward, "Low-noise mems vibration sensor for geophysical applications," J. Microelectromech. Syst., vol. 8, no. 4, pp. 433-438, Apr. 1999.

[7] E. Peiner, D. Scholz, K. Fricke, A. Schlachetzki, and P. Hauptmann, "Microelectromechanical vibration sensor with optical interconnects," J. Microelectromech. Syst., vol. 7, no. 1, pp. 56-61, Jan. 1998.

[8] D. Haronian, "Geometrical modulation-based interferometry for displacement sensing using optically coupled suspended waveguides," $J$. Microelectromech. Syst., vol. 7, no. 3, pp. 309-314, Mar. 1998.

[9] E. Ollier, P. Philippe, C. Charbol, and P. Mottier, "Micro-optomechanical vibration sensor integrated on silicon," J. Microelectromech. Syst., vol. 17, no. 1, pp. 26-29, Jan. 1999.

[10] A. Selvarajan, P. K. Pattnaik, V. M. Gupta, and T. Srinivas, "Micro-opto -electro-mechanical (MOEM) vibration sensor," in Proc. of SPIE, vol. 3990, Singapore, Mar. 2000, pp. 78-85.

[11] B. E. Little, S. T. Chu, H. A. Haus, J. Foresi, and J. P. Laine, "Microring resonator channel droping filters," J. Lightwave Technol., vol. 15, no. 6 , pp. 998-1005, June 1997.

[12] A. Melloni, R. Costa, P. Monguzzi, F. Morichetti, G. Cusmai, F. Morichetti, and M. Martinelli, "Experimental investigation on ring resonators based filters in sion technology," in Proc. of 11th European Conference on Integrated Optics(ECIO), vol. 1, Prague, Czech Republic, Apr. 2003, pp. 375-378.

[13] A. Lovacs and Z. Vizvary, "Structural parameter sensitivity analysis of cantilever- and bridge-type accelerometers," Sensors and Actuators A, vol. 89, pp. 197-205, 2001 